\title{
Philonsorbonne
}

12 | 2018

Année 2017-2018

\section{Philosophus non supra grammaticam. La grammaire philosophique de Rosenzweig}

\section{Émeline DURAND}

\section{(2) OpenEdition \\ Journals}

Édition électronique

URL : https://journals.openedition.org/philonsorbonne/954

DOI : 10.4000/philonsorbonne.954

ISSN : 2270-7336

Éditeur

Publications de la Sorbonne

\section{Édition imprimée}

Date de publication : 1 janvier 2018

Pagination : 27-44

ISSN : 1255-183X

\section{Référence électronique}

Émeline DURAND, «Philosophus non supra grammaticam. La grammaire philosophique de

Rosenzweig ", Philonsorbonne [En ligne], 12 | 2018, mis en ligne le 03 mars 2018, consulté le 08 juin 2021. URL : http://journals.openedition.org/philonsorbonne/954 ; DOI : https://doi.org/10.4000/ philonsorbonne.954 


\title{
Philosophus non supra grammaticam. La grammaire philosophique de Rosenzweig
}

\author{
Emeline DURAND
}

Franz Rosenzweig (1886-1929), philosophe allemand de confession juive, est l'auteur d'une singulière réflexion sur le langage. Dans son grand œuvre L'Étoile de la Rédemption (1921), il prend le contrepied de la tradition philosophique, qui pèche selon lui par son indifférence voire sa méfiance à l'égard du langage. En réaction à l'idéalisme allemand, dont Rosenzweig affirme qu'il "n'a pas de philosophie du langage »", il propose une critique et une refondation de la philosophie sous la forme d'une "pensée parlante » ou pensée du langage (Sprachdenken). Dès lors, aucun fait de langue ne saurait être étranger à l'interrogation philosophique : les analyses proposées dans l'Étoile théorisent la relation entre le mot et la chose, s'appuient sur l'observation de la morphologie et de la syntaxe de certaines langues, réfléchissent à la dimension pragmatique, et non pas seulement représentationnelle, du langage.

La grammaire joue un rôle central dans cette méthode philosophique, que Rosenzweig, dans l'essai de 1925 «La pensée nouvelle», qualifie de «pensée grammaticale», en l'opposant à la «pensée logique» de l'idéalisme ${ }^{2}$. Dans le même texte, il revendique son appartenance à une famille de pensée qui, de Feuerbach à Hermann Cohen puis à ses

\footnotetext{
1. Franz Rosenzweig, Die “Gritli”-Briefe: Briefe an Margrit Rosenstock-Huessy, éd. Inken Rühle et Reinhold Mayer; préface de Rafael Rosenzweig, Tübingen, Bilam-Verlag, 2002 (désormais Gritli-Briefe), p. 144.

2. Franz Rosenzweig, Foi et savoir : autour de L'Étoile de la Rédemption, textes introduits, traduits et annotés par Gérard Bensussan, Marc Crépon et Marc de Launay, Paris, Vrin, 2001 (désormais Foi et savoir), p. 159 ; Der Mensch und sein Werk: Gesammelte Schriften, III : Zweistromland, éd. Reinhold et Annemarie Mayer, La Haye, M. Nijhoff, 1982 (désormais GS III), p. 151.
} 
contemporains Rosenstock-Huessy, Weizsäcker et Ehrenberg, fait le choix d'interroger le réel depuis la grammaticité du langage. Pour comprendre la pensée de Rosenzweig, il est donc essentiel d'élucider ce qu'il entend par grammaire. En quoi la «pensée parlante» est-elle une "pensée grammaticale»? Que signifie mener une rigoureuse critique de la philosophie depuis le point de vue de la grammaire?

S'il est généralement admis que Rosenzweig doit son intérêt pour la grammaire à l'influence de son ami Eugen Rosenstock-Huessy ${ }^{3}$, tout ce que Rosenzweig hérite de la philosophie allemande du langage est en revanche souvent passé sous silence, comme l'a souligné Donatella Di Cesare ${ }^{4}$. C'est le double héritage de la grammaire générale et de la grammaire comparée que l'on voudrait contribuer à mettre en lumière.

«Le philosophe n'est pas au-dessus de la grammaire » : cette remarque en latin dans le texte, variation sur le proverbial "Caesar non supra grammaticos » du concile de Constance, résume le ton de cette méthode qui invite la philosophie à se mettre à l'écoute de la grammaire ${ }^{5}$. Cependant, Rosenzweig ajoute aussitôt dans le même texte : « Mais pourquoi ne devraitil pas y avoir de grammaires antiphilosophiques? ». Cette expression, qui demande à être élucidée dans son contexte, jette le soupçon sur l'identification pure et simple entre la méthode grammaticale et la "pensée nouvelle »: elle rappelle qu'il existe à tout le moins des grammaires qui ne se plient pas à ce que la philosophie peut attendre d'elles. Se pose dès lors la question du rapport entre la philosophie et les langues naturelles en leur diversité.

On montrera (I) que le recours à la grammaire se comprend, chez Rosenzweig, dans un cadre critique : la polémique contre le panlogisme hégélien l'amène à réinvestir le champ grammatical. Au-delà de cette dimension critique, on proposera (II) de voir en L'Étoile de la Rédemption une contribution originale au projet classique de grammaire générale. On s'interrogera enfin (III) sur la thèse fondamentale de la grammaire

3. Sur le rapport entre les pensées de Rosenzweig et de Rosenstock-Huessy sur le langage, voir Adam Zak, Vom reinen Denken zur Sprachvernunft. Über die Grundmotive der Offenbarungsphilosophie Franz Rosenzweigs, Stuttgart, W. Kohlhammer, 1987, en particulier p. 29-54; Martin Brasser, "Rosenstock und Rosenzweig über Sprache. Die Angewandte Seelenkunde im Stern der Erlösung ", in Martin Brasser(éd.), Rosenzweig als Leser. Kontextuelle Kommentare zum Stern der Erlösung, Tübingen: Niemeyer, 2004, p. 173-207.

4. Donatella Di Cesare, «L'expression du duel dans la Rédemption. À propos de la généalogie du Nous chez Rosenzweig », in Myriam Bienenstock (éd.), Héritages de Franz Rosenzweig, Éditions de l'Éclat, "Bibliothèque des fondations », 2011, p. 114: «En le [sc. : Rosenzweig] séparant ainsi de la tradition "classique" de la philosophie du langage, on passe à côté de son originalité qui consiste en ce qu'il emprunte les éléments existants, les réinterprète selon le cas pour en faire les fondements de son "nouveau système" ".

5. La place manque pour approfondir ce point, mais il est possible que ce propos réponde aussi à celui de Nietzsche : «Le philosophe n'aurait-il pas le droit de s'élever au-dessus de la foi qui régit la grammaire?»(Par-delà bien et mal, trad. Cornélius Heim, Paris, Gallimard/Folio, 2002, p. 54). 
rosenzweigienne, celle d'une unité du langage par-delà la diversité des langues, dont on proposera de reconstruire la double formulation linguistique et théologique.

\section{I}

L'intérêt de Rosenzweig pour la grammaire apparaît sur le fond du panlogisme qu'il associe à l'idéalisme allemand, essentiellement dans sa version hégélienne. La grammaire est l'instrument de ce qu'on peut appeler la critique linguistique (Sprachkritik) de l'idéalisme : une critique menée au nom du langage et par le moyen du langage. À ce titre, L'Étoile de la Rédemption semble mettre en scène une opposition irréconciliable entre grammaire et logique.

Rosenzweig accepte la définition hégélienne de la logique comme « science de l'Idée pure, c'est-à-dire de l'Idée dans l'élément abstrait de la pensée $»^{6}$. Il lui oppose la grammaire comme "science des sons vivants», qui se divise en l'étude des parties du discours («théorie des formes lexicales ») et celle des règles qui donnent forme à la langue (« légalité régissant la totalité du langage $\left.{ }^{7} \gg\right)$. Cette opposition ne tient manifestement aucun compte des développements que Hegel a lui-même consacrés à la grammaire et à sa similarité avec la logique, de l'introduction à la Science de la logique au $\S 459$ de l'Encyclopédie ${ }^{8}$. La présentation que l'on va lire ne cherche pas à nier la dimension d'injustice ou de malentendu que comporte la critique rosenzweigienne, mais plutôt à en retracer les motivations dans le projet d'ensemble de l'Étoile.

Lorsque la grammaire entre en scène, dans la deuxième partie de l'Étoile, elle est d'emblée porteuse d'une charge critique à l'égard de la logique idéaliste, accusée d'être l'instrument d'une dépréciation du langage. Pour Rosenzweig, la pensée idéaliste porte le soupçon sur la capacité du langage à exprimer les pensées et le réel, et veut lui substituer un mode de pensée, le savoir absolu, qui prétend s'élever au-dessus du caractère contingent et limité du langage ${ }^{9}$. Cette exigence conduit l'idéalisme à un geste que Rosenzweig présente comme une folie : le développement d'une méthode logique autonome, dont la rupture avec le langage se traduit par le

6. G.W.F. Hegel, Encyclopédie des sciences philosophiques, I. La science de la logique, § 19, trad. B. Bourgeois, Paris, Vrin, 1970, p. 283.

7. Franz Rosenzweig, L'Étoile de la Rédemption, trad. Alexandre Derczanski et Jean-Louis Schlegel (nouvelle édition), Paris, Seuil, 2003 (désormais Étoile), p. 182 (trad. modifiée); Der Stern der Erlösung, Frankfurt am Main, Suhrkamp, 2015 (désormais Stern), p. 139-140.

8. Sur la grammaire chez Hegel, voir notamment Jim Vernon, « The Realm of Abstraction: The Role of Grammar in Hegel's Linguistic System », in Jere O'Neill Surber (éd.), Hegel and Language, Albany: State University of New York Press, 2006, p. 165-177.

9. Étoile p. 209, Stern p. 161. 
fait qu'elle se veut « indépendante par rapport à la grammaire ». L'idéalisme s'en remet à une logique «pure», «ennemie de la langue» (eine sprachfeindliche Logik), "étrangère » à elle, donc finalement « en retrait de $1^{\prime}$ homme ${ }^{10} »$. Un exemple en est fourni par le processus logique grâce auquel l'idéalisme (et bien que Rosenzweig ne nomme pas son adversaire, on peut penser que c'est ici plutôt Fichte qui est visé) entend passer du Moi au NonMoi, c'est-à-dire d'un sujet à une objectivité. Grammaticalement, ce passage devrait être celui d'un pronom (« Je ») à un ensemble d'adjectifs énumérant les qualités objectives du monde qui fait face au Je. Or pour Rosenzweig, une telle connexion est contraire à la grammaire : un adjectif ne saurait être déduit d'un pronom. Là où Hegel critiquait la conception fichtéenne parce qu'elle présuppose le caractère absolu du Moi et du Non-Moi et de leur séparation $^{11}$, Rosenzweig formule sa critique de la dialectique en les termes d'une impossibilité grammaticale. Si elle ne respecte pas l'ordre de la grammaire, la connexion invoquée par l'idéalisme n'a d'autre option que d'être "purement logique »; son importance fondatrice au cœur du système met le langage en échec dès le premier instant ${ }^{12}$.

Si la grammaire des langues naturelles est la victime des assauts logiques de l'idéalisme, c'est elle aussi qui sera le point de départ d'une contre-offensive. Il faut en effet venir contester à la « logique ennemie de la langue » son hégémonie dans la pensée philosophique. Rosenzweig le fait au nom d'une "pensée grammaticale », qui s'en remet au langage pour comprendre deux aspects essentiels du réel auxquels l'idéalisme demeure étranger : la temporalité et l'ouverture à l'altérité ${ }^{13}$.

On ne peut toutefois en rester à cette lecture qui fige les notions de grammaire et de logique en une opposition statique. Le système de philosophie proposé dans l'Étoile se caractérise au contraire par son dynamisme, sa tendance à reconsidérer sous une nouvelle lumière les relations établies en première approche. Dans l'introduction à la deuxième partie de l'Étoile, Rosenzweig commence certes par opposer une « langue de la logique » (jene Sprache der Logik) et la « langue réelle de la grammaire » (einer wirklichen Sprache der Grammatik). Il donne bien sûr la priorité à la seconde, disant même de la première qu'elle n'est pas véritablement une langue, mais seulement un ensemble d'éléments muets et cachés, formant tout au plus « un langage d'avant le langage ». Il nous invite à quitter les muettes relations logiques explorées par l'idéalisme pour nous ouvrir au langage vivant, tel qu'il est parlé par des locuteurs. Mais dès le paragraphe suivant, l'opposition est reformulée en une relation étroite " entre la logique $\mathrm{du}$ langage et sa grammaire» (in diesem Verhältnis zwischen der Logik der Sprache und ihrer Grammatik). Rosenzweig affirme même que c'est

10. Étoile p. 203-204, Stern p. 155-157.

11. G.W.F. Hegel, Encyclopédie des sciences philosophiques, III. Philosophie de l'esprit, $\S 415$, Addendum, trad. B. Bourgeois, Paris, Vrin, 1988, p. 521.

12. Étoile p. 203, Stern p. 156-157.

13. Foi et savoir, p. 159. 
dans cette relation que se trouve la clé de tout l'ouvrage, permettant de comprendre d'autres articulations fondamentales de son système - la relation entre Création et Révélation, entre philosophie et théologie ${ }^{14}$. On est passé en quelques lignes d'une Sprache der Logik, hostile à la grammaire, à une Logik der Sprache, compatible avec et complémentaire à la grammaire. Loin d'être érigée en opposition massive, la distinction entre logique et grammaire est reportée à l'intérieur de l'étude du langage. Dans une note plus tardive sur Herder, Rosenzweig emploiera l'expression de logique de la langue (Sprachlogik) et dira qu'elle est seule en mesure de nous mettre sur la voie d'une véritable compréhension de la nature du langage ${ }^{15}$. Autrement dit, la découverte d'une authentique philosophie du langage, qui faisait défaut à l'idéalisme allemand, ne repose pas sur une critique unilatérale de la logique, mais sur une reconceptualisation des relations entre logique et grammaire, qui constituent les deux aspects complémentaires de cette nouvelle approche.

Ces remarques suggèrent qu'une relecture des analyses de l'Étoile s'impose, qui détermine ce qui est véritablement en jeu, au-delà de la polémique avec l'idéalisme, dans la notion rosenzweigienne de grammaire.

\section{II}

La solidarité entre la logique et la grammaire comme Sprachlogik rappelle les grammaires philosophiques des $\mathrm{XVII}^{\mathrm{e}}$ et XVIII ${ }^{\mathrm{e}}$ siècles, pour qui la syntaxe tient lieu de logique de la langue. Le rapprochement des termes de la langue avec des opérations de l'esprit permet de procéder à la déduction des parties du discours plutôt qu'à leur collection empirique. L'on cherche ainsi à dégager une grammaire qui serait le modèle de toute langue, de même que la logique est le modèle de toute pensée. En Allemagne, le projet de grammaire philosophique n'a pas disparu avec le criticisme ni avec l'idéalisme. Comme l'a montré Raphaël Ehrsam, Kant considère encore la grammaire générale comme une science légitime, ayant sa place dans le projet transcendantal, bien qu'il ne lui ait guère consacré de développement explicite $^{16}$. Une grammaire philosophique d'inspiration kantienne voit le jour avec la Théorie du langage d'August Ferdinand Bernhardi ${ }^{17}$.

L'Étoile de la Rédemption apporte à l'histoire de la grammaire philosophique une contribution singulière et inattendue. En septembre 1918, Rosenzweig envoie à son cousin Rudolf Ehrenberg le plan détaillé de

14. Étoile p. 160-161, Stern p. 121-122.

15. Franz Rosenzweig, Der Mensch und sein Werk: Gesammelte Schriften, I.2 : Briefe und Tagebücher 1918-1929, éd. R. Rosenzweig et E. Rosenzweig-Scheinmann; avec la participation de Bernhard Casper, La Haye, M. Nijhoff, 1979 (désormais GS I.2), p. 774.

16. Raphaël Ehrsam, Le Problème du langage chez Kant, Paris, Vrin, 2016, p. 101-142.

17. Voir Denis Thouard, Et toute langue est étrangère. Le projet de Humboldt, Paris, Les Belles Lettres, 2016, p. 79-115. 
l'ouvrage qu'il projette, accompagné du commentaire suivant: «La théorie $\mathrm{du}$ langage [Sprachlehre] et la philosophie de l'art sont présentes tout au long de l'ouvrage ${ }^{18}$. Il attire ainsi notre attention sur le fait que la réflexion sur le langage n'a pas de lieu propre dans l'Étoile: elle ne saurait être contenue dans un chapitre ni constituer un moment du système, mais elle fournit à l'ensemble son inspiration. Le choix du terme de Sprachlehre est une référence discrète à une certaine manière d'étudier le langage : cela pourrait être le titre d'un manuel pour l'étude d'une langue particulière, mais aussi celui d'un traité de grammaire générale, comme c'est le cas par exemple chez Bernhardi (Reine Sprachlehre, Berlin, 1801-1803) et J. S. Vater (Versuch einer allgemeinen Sprachlehre, Halle, 1801). L'adoption de cette terminologie par Rosenzweig ne suffit certes pas à parler d'une reprise explicite du projet de grammaire philosophique, mais elle indique une préoccupation commune. Le terme de Sprachlehre apparaît d'ailleurs dans l'Étoile, lorsque Rosenzweig réfléchit sur l'ordre d'exposition des catégories grammaticales ${ }^{19}$. S'il est évident qu'on ne peut pas réduire l'Étoile - un livre qui en contient plusieurs - à un traité sur le langage, il est possible d'interpréter ses trois parties comme trois niveaux de grammaire spéculative.

La première partie de l'Étoile («Les éléments ou le perpétuel prémonde $»)$ descend au niveau le plus fondamental et pose la question de savoir quelles sont les conditions nécessaires pour qu'existe un langage en général. Rosenzweig répond que tout langage repose sur trois opérations essentielles, qui sont l'affirmation, la négation et la conjonction ${ }^{20}$. Partout où des individus affirment, nient et articulent des idées, il y a langage. Ces opérations, étant fondamentales pour le langage en général, ont aussi une traduction concrète dans toute langue particulière, qui comporte une manière d'affirmer (« oui »), de nier (« non »), de conjoindre (« et»). Ainsi pourraient s'expliquer les mystérieux Urworte que Rosenzweig fait entrer en scène dans cette première partie et qui posent un problème d'interprétation majeur au lecteur de l'Étoile. Rosenzweig emprunte ces trois mots au langage réel, c'est-à-dire à une langue déterminée, l'allemand, pour nommer les trois opérations qu'il inscrit au cœur du langage en général. L'expression de Urwort signifie sans doute moins un «mot-origine », comme le propose la traduction française, qu'une opération constitutive de la parole ${ }^{21}$.

Parler d'individus (qui affirment, nient et conjoignent) est paradoxal et procède d'une anticipation sur les analyses de la deuxième partie. En effet,

18. GS I.2, p. 605. Voir également la lettre envoyée le même jour à Margrit RosenstockHuessy, Gritli-Briefe, p. 144.

19. Étoile p. 216, Stern p. 167.

20. Voir la lettre à Margrit Rosenstock-Huessy du $1^{\mathrm{er}}$ octobre 1918, Gritli-Briefe p. 156 : "Wie ja auch die Sprache hier noch nicht da ist, sondern nur ihre Elemente (das Bejahen, das Verneinen, das Verbinden) ».

21. Barbara E. Galli, auteur en 2005 d'une nouvelle traduction anglaise de l'Étoile, traduit cette expression par «original word» (The Star of Redemption, Madisonn The University of Wisconsin Press, 2005, p. 35). 
du point de vue purement conceptuel qui est ici celui de Rosenzweig, il n'y a pas encore de locuteurs : Dieu, l'homme et le monde sont présentés comme des êtres entièrement muets. Ce sont les conditions de possibilité purement pensées du langage qui sont dévoilées. En tant que telles, ces conditions de possibilité sont inaudibles ${ }^{22}$ : elles peuvent tout au mieux être représentées visuellement par un système d'équations, qui constitue l'unique concession de Rosenzweig à un certain langage formel. En ce sens, on peut dire que la grammaire philosophique de l'Étoile commence par une logique du langage. Celle-ci n'est plus une « logique ennemie de la langue» : loin d'isoler l'art de penser de celui de parler, elle étudie les opérations de pensée que sont l'affirmation, la négation et la conjonction en vue de la manière dont nous parlons. Telle est la première leçon de la Sprachlogik : c'est parce qu'il y a une communauté, voire une universalité du penser, que les locuteurs peuvent se comprendre dans le langage ${ }^{23}$. L'étude de ces opérations pré-linguistiques permet d'aboutir à l'idée d'une «raison dans la langue » (Sprachvernunft), qui, récusant tout savoir absolu, vient nommer l'intrication de la pensée et des mots dans la production du discours ${ }^{24}$.

La deuxième partie de l'ouvrage (« La voie ou le monde constamment renouvelé ») amène un complet changement de perspective : les locuteurs entrent en scène, le langage devient effectif (wirkliche Sprache). Dans l'idiome théologique de l'Étoile, cela signifie que Dieu, le monde et l'homme abandonnent leur mutisme initial et se mettent à parler. Dès lors, les opérations fondamentales identifiées dans la première partie perdent leur statut de Urworte imprononçables et se réalisent dans des tournures grammaticales particulières. Rosenzweig suit les principes de la grammaire générale en proposant une analyse détaillée des parties du discours qui, si elle fait appel ici et là à des formes spécifiques à telle ou telle langue, est bien censée valoir pour toutes les langues. Du niveau abstrait du langage en général, on est passé à l'universalité concrète des langues, étudiées du point de vue de leur commune grammaticité.

Rosenzweig est confronté à la nécessité de choisir un certain ordre dans sa présentation des parties du discours. Sa position est claire : il ne s'agit pas de déduire les catégories les unes des autres, de telle sorte qu'on ne puisse revenir en arrière dans l'ordre proposé. Chaque catégorie grammaticale présuppose l'existence simultanée de toutes les autres, ce qui interdit le recours à une présentation généalogique. La présentation n'est pas non plus systématique au sens où elle suivrait un chemin unique et nécessaire: Rosenzweig veut préserver la possibilité de recommencer l'exposition grammaticale en fonction de la nature du langage étudié, donc de faire intervenir une même catégorie grammaticale à plusieurs reprises en

22. Étoile p. 160, Stern p. 121.

23. Étoile p. 160-161 (trad. modifiée), Stern p. 121-122: « [...] en chaque individu pour soi, la pensée est muette et pourtant commune à tous ; à travers cette communauté, la pensée fonde la véritable communauté de la parole [...]».

24. Étoile p. 59, Stern p. 36. 
lui attribuant une fonction différente. Sa thèse fondamentale, en effet, est que l'ordre grammatical n'est pas immanent au langage, mais lui est conféré depuis l'extérieur : il reflète la relation entre le langage et la réalité ${ }^{25}$.

$\mathrm{Ce}$ vocabulaire traduit une influence kantienne: la grammaire se propose d'examiner la manière dont les catégories linguistiques se rapportent au réel, c'est-à-dire de procéder à une déduction des catégories, non pas au sens génétique (comme le fait par exemple Fichte dans l'essai de 1795 sur la faculté linguistique ${ }^{26}$ ), mais au sens qu'a cette expression dans la Critique de la raison pure. La piste kantienne se confirme lorsque Rosenzweig montre comment l'on passe du Urwort inaudible à un premier mot audible, le Stammwort («mot-souche»), qui hérite du Urwort sa valeur affirmative, négative ou conjonctive, mais joue aussi le rôle de paradigme duquel dérivent ensuite les expressions concrètes du langage. Rosenzweig écrit alors que le Stammwort se « schématise » en différentes formes linguistiques ${ }^{27}$.

Tels sont les principes qui dictent à Rosenzweig la méthode, ou plutôt les méthodes, de sa grammaire. Les concepts de la théologie permettent de distinguer les trois modalités sous lesquelles le réel se donne à nous : l'événement (Ereignis), qui correspond à la Création; l'expérience vécue (Erlebnis) de la Révélation; l'anticipation (Vorwegnahme), par laquelle nous nous rapportons dès maintenant à la Rédemption à venir. Le langage traduit ces trois processus de manière distincte, ce qui justifie que, pour chacun, l'on recommence l'exposition grammaticale ${ }^{28}$.

Dans le chapitre consacré à la Création, la déduction des catégories grammaticales vise à déterminer comment le langage peut se rapporter à des choses en tant qu'elles interviennent dans un événement objectif qui pourra faire l'objet d'un récit. L'ordre de présentation montre comment on passe de la pure qualité (exprimée par l'adjectif) à la choséité accomplie (exprimée par le substantif et sa détermination), puis à la mise en rapport des choses dans des événements (exprimés par les formes verbales). L'explication grammaticale se concentre sur le langage en tant qu'il est capable de présenter une image objective du monde, grâce aux formes linguistiques caractéristiques du récit: le mode indicatif, le temps du passé, la troisième personne du singulier.

Le chapitre consacré à la Révélation se penche sur la manière dont le langage peut traduire une expérience vécue qui se déroule sous la forme d'un dialogue entre deux sujets, puisque la Révélation nomme chez Rosenzweig un échange de paroles entre Dieu et l'homme. La méthode grammaticale ne peut plus consister, comme pour la Création, à passer d'une catégorie

25. Étoile p. 182-183, Stern p. 140.

26. J. G. Fichte, «De la faculté linguistique et de l'origine du langage », in Essais philosophiques choisis : 1794-1795, trad. Luc Ferry et Alain Renaut, Paris, Vrin, 1984.

27. Étoile p. 185, Stern p. 142 : «Gehen wir nun vom Stammwort zur Schematisierung der Wortformen über $»$.

28. Étoile p. 216, Stern p. 167. 
linguistique à une autre. Elle suit les étapes de ce dialogue en traitant les paroles échangées entre Dieu et l'homme comme autant d'actes de parole : appel et réponse, commandement, aveu (aveu d'amour devenant confession des péchés puis confession de foi), prière, cri. Sont ainsi mises en lumière les catégories grammaticales qui permettent l'expression de la subjectivité et de l'intersubjectivité dans le langage : les pronoms Je et $\mathrm{Tu}$, le nom propre ; ainsi que les formes verbales qui traduisent le pur présent de l'expérience vécue : l'aspect inaccompli des verbes, l'impératif.

Enfin, le chapitre consacré à la Rédemption dévoile la dimension lyrique du langage, par laquelle l'éternité est anticipée dans l'instant présent. La parole ici n'est plus un récit successif, ni un dialogue, mais la réunion de voix distinctes en une parole commune, le chant de louange. La méthode grammaticale a pour tâche de montrer comment toutes les formes linguistiques convergent pour exprimer un sens unique, lui-même porteur d'une fonction rédemptrice. Les catégories grammaticales concernées sont celles qui explicitent la relation de l'homme au monde, relation essentielle pour l'advenue de la Rédemption. Il s'agit de montrer que le langage porte la trace du prochain, à travers les pronoms personnels Nous et Vous. Rosenzweig consacre aussi une analyse à la forme du duel, caractéristique de certaines langues qui distinguent le simple pluriel des choses et la multiplicité des personnes : le duel est le creuset de l'intersubjectivitée ${ }^{29}$. Le futur, porteur de l'anticipation de la Rédemption dans le langage, est le temps privilégié ici.

De cette triple méthode grammaticale, on peut tirer la conclusion suivante. En plaçant sa grammaire générale sous les notions de Création, de Révélation et de Rédemption, Rosenzweig ne fait pas que développer l'idiome théologique qui caractérise le discours de l'Étoile: il innove en soutenant que l'étude des parties du discours doit être liée à celle du contexte dans lequel les mots sont employés. Il n'est pas loin, à ce titre, d'une approche pragmatique du langage. La Création, la Révélation et la Rédemption fournissent à l'analyse grammaticale sa matière, sous la forme d'un répertoire de formes lexicales ${ }^{30}$ et du contexte de leur emploi. La grammaire de Rosenzweig n'est pas seulement une étude détaillée des parties du discours, elle est aussi une tentative pour rendre compte de la logique propre des propos dans lesquels elles figurent. Cela explique que l'analyse grammaticale se structure, dans chaque chapitre de cette partie centrale, en trois temps : les catégories linguistiques concernées sont d'abord passées en revue (c'est ce que Rosenzweig appelle Grammatik), puis les relations entre elles sont interprétées philosophiquement (c'est la tâche de la Logik), avant que la lecture d'un passage du texte biblique n'illustre la façon

\footnotetext{
29. Sur l'approche rosenzweigienne du duel et sa proximité avec les idées de Humboldt, voir l'article déjà cité de Donatella Di Cesare, «L'expression du duel dans la Rédemption. À propos de la généalogie du Nous chez Rosenzweig », in Myriam Bienenstock (éd.), Héritages de Franz Rosenzweig, Éditions de l'Éclat, « Bibliothèque des fondations », 2011, p. 114-123.

30. Étoile p. 220, Stern p. 170 («Wortformenschatz»).
} 
dont ces relations sont réalisées dans le langage (c'est le rôle de l'analyse grammaticale, grammatische Analyse, qui conclut chaque chapitre).

La grammaire de Rosenzweig ne prétend donc pas être une « science du langage » dotée d'un objet propre qu'elle serait seule à posséder ${ }^{31}$. Parce qu'elle comporte une dimension pragmatique, elle s'ouvre aux apports de la philosophie, qui lui fournit les catégories logiques dont elle a besoin, et de l'exégèse, qui lui donne accès aux textes dans lesquels se reflète par excellence la grammaticité du langage. À ce titre, la grammaire générale de Rosenzweig est en même temps une théologie du langage, au sens spécifique qu'a ce terme dans l'Étoile. Dans "La pensée nouvelle », Rosenzweig fait d'ailleurs reposer la dimension théologique de l'Étoile - sa capacité à traduire les problèmes théologiques en problèmes humains et à élever les problèmes humains jusqu'à une expression théologique - sur la collaboration de la grammaire et de la logique, destinée à (re)mettre le langage au cœur de la philosophie ${ }^{32}$.

\section{III}

On s'attendrait à ce que, dans la troisième partie de l'Étoile (« La figure ou le sur-monde éternel »), Rosenzweig abandonne le niveau de l'universel grammatical pour évoquer de plus près les singularités des langues, en adoptant par exemple une démarche comparative. Nous savons que la grammaire comparée était connue de lui : en 1916-1917, alors qu'il mûrissait les idées qui donneraient naissance à l'Étoile, il a lu avec beaucoup d'intérêt l'ouvrage du linguiste Franz Nikolaus Finck intitulé Die Haupttypen des Sprachbaus, publié à Leipzig en $1909^{33}$. Dans l'Étoile, pourtant, le projet de revenir au niveau de la diversité des langues est absent. Les seules allusions à une langue en particulier concernent l'hébreu, qui est en outre abordé non plus sous l'angle grammatical, mais sous l'angle théologico-politique, en tant que langue du peuple juif. Plutôt que de s'engager dans une grammaire comparée, la troisième partie de l'Étoile prolonge la dimension pragmatique en direction d'une sociologie du langage: Rosenzweig se penche sur la

31. Étoile p. 216, Stern p. 167.

32. Foi et savoir, p. 161 ; GS III, p. 153 : «Les problèmes théologiques veulent être traduits en problèmes humains et les problèmes humains veulent s'élever jusqu'à une expression théologique. [...] Les problèmes de la pensée traditionnelle sont pour la plupart tout simplement inaperçus, et lorsqu'ils se font jour, ils ne sont pas reconnus comme tels ; cela ne vaut pas seulement pour les questions théologiques au sens restreint, mais aussi pour la majorité des problèmes humains que la méthode grammaticale permet d'appréhender de manière rigoureuse, grâce à la logique du je et tu, par exemple, ou grâce à la logique des noms $[\ldots] »$ (Je souligne).

33. Voir Franz Rosenzweig, Der Mensch und sein Werk: Gesammelte Schriften, I.1 : Briefe und Tagebücher 1900-1918, éd. R. Rosenzweig et E. Rosenzweig-Scheinmann avec la participation de Bernhard Casper, La Haye, M. Nijhoff, 1979 (désormais GS I.1), p. 272. 
relation des communautés juive et chrétienne à la parole. Il s'agit d'ailleurs plutôt d'une sociologie du silence, qui n'est pas négation du langage, mais accomplissement de la compréhension entre les hommes et préparation à la vie de la communauté ${ }^{34}$.

On peut s'interroger sur les raisons de ce choix d'en rester à l'approche spéculative. Le projet grammatical de Rosenzweig demeure-t-il inachevé s'il lui manque la prise en compte de la diversité des langues? Préserver le caractère philosophique de la grammaire exigeait-il au contraire de laisser de côté cette diversité pour mieux rendre compte de l'unité du langage?

La réponse à cette question implique de se pencher sur la thèse qui constitue la clé de voûte de toute la pensée rosenzweigienne du langage. L'Étoile en donne une formulation paradoxale: "Il se peut qu'il existe plusieurs langages [ou plusieurs langues], mais il n'y a qu'un seul langage [qu'une seule langue ${ }^{35}$ ] » (es kann zwar viele Sprachen geben, aber nur eine Sprache). Dans la préface à sa traduction des poèmes de Juda Halévy (1924), Rosenzweig écrit plus directement : «Il n'y a qu'une seule langue » (Es gibt nur Eine Sprache $\left.{ }^{36}\right)$. De l'Étoile aux textes ultérieurs sur la traduction, ce motif est donc répété avec une grande constance. Il ne vise pas à réduire la diversité des langues, mais au contraire à la rendre féconde dans l'unité d'un même langage et d'une même Parole.

Dès l'époque de ses premières lectures autour de la grammaire comparée, en 1916, Rosenzweig se montre conscient de la nécessité de ne pas s'en tenir à l'infinie diversité du phénomène linguistique dans le monde. En témoigne une brève note sur Humboldt qui soupçonne celui-ci d'avoir sciemment érigé «l'infinité empirique des langues» en objet d'étude, au point que celle-ci fait écran, l'empêche d'accomplir sa véritable tâche philosophique ${ }^{37}$. Rosenzweig ne dit pas quelle est cette tâche. On sait que la sienne propre consiste, sous le nom de Sprachdenken, à s'appuyer sur le langage pour donner à la philosophie un souffle nouveau. Le motif de l'unité $\mathrm{du}$ langage est une pièce importante de ce projet, en tant qu'il a le double statut d'une thèse linguistique et d'un théologoumène.

La dimension proprement linguistique est illustrée par un texte antérieur à l'Étoile, l'essai programmatique «Volksschule und Reichsschule »

34. Voir par exemple Étoile p. 440-441, Stern p. 350-351.

35. Étoile p. 212, Stern p. 164. La traduction française se trouve confrontée à la nécessité de choisir entre les termes de langue et de langage, là où l'allemand joue sur le double emploi de Sprache.

36. Franz Rosenzweig, Der Mensch und sein Werk: Gesammelte Schriften, IV : Sprachdenken im Übersetzen. Vol. 1: Jehuda Halevi. Fünfundneunzig Hymnen und Gedichte, éd. R. Rosenzweig, La Haye, M. Nijhoff, 1983 (désormais GS IV.1), p. 3 ; Franz Rosenzweig, L'Écriture, le verbe et autres essais, trad. Jean-Luc Évard, Paris, PUF, 1998, p. 156.

37. GS III, p. 69 : «Ob Humboldt (Wilhelm) nicht sehr gern die empirische Unendlichkeit der Sprachen zwischen sein Individuum und seine philosophische Aufgabe als einen Puffer zwischen eingeschaltet hat? Der Verdacht liegt nahe». 
(«École populaire et école impériale », $1916^{38}$ ). La réflexion pédagogique de Rosenzweig est orientée vers l'idéal d'une unité culturelle de la nation. L'éducation qu'il propose obéit à ce principe d'unité contre la traditionnelle division en disciplines. L'accent y est mis sur le développement de trois capacités fondamentales, qui font de l'école une véritable préparation à la vie : une conscience historique approfondie ; une technique mathématisée permettant la maîtrise de la nature ; la pratique vivante de la diversité des langues.

La réorganisation des savoirs proposée dans la section consacrée aux langues (Sprachenkunde) vise d'abord à résoudre une difficulté pratique : dans le système que Rosenzweig connait, les élèves consacrent plusieurs années à apprendre une langue vivante, sans que cette étude leur permette, en règle générale, de la parler à l'âge adulte. L'apprentissage isolé d'une langue conduit en outre à une certaine instabilité dans le rapport de l'élève à sa langue maternelle. Les écoles allemandes forment des individus dont la conscience linguistique est à moitié éveillée, incapables de sortir de l'état d' " entre-deux-langues » qui est le leur. C'est là une fort mauvaise préparation à la rencontre des autres cultures ${ }^{39}$.

Rosenzweig propose d'instaurer l'apprentissage, non plus d'une ou plusieurs langues en tant que telles (l'italien, le français, le grec), mais du système des rapports qui unissent les langues humaines entre elles: c'est la seule manière de rendre l'élève capable d'apprendre intelligemment n'importe quelle langue européenne, mais aussi de s'initier à la diversité des langues de la planète. La grammaire comparée fournit sa méthode à ce programme ambitieux, qui comprend l'étude des langues germaniques, la comparaison entre les langues indo-européennes, puis l'initiation à quelquesuns des grands types linguistiques (sémitique, chinois, finno-ougrien, bantou, eskimo). L'approche comparative permet de s'élever de la "lettre » à «l'esprit» du langage, c'est-à-dire de la grammaire de chaque langue singulière à l'essence du langage, commune à toutes les langues ${ }^{40}$.

Ce programme d'enseignement arrive à son terme avec la dernière année d'école, consacrée à la philosophie, bien que ce «nom grandiloquent» doive être évité. Sous le nom de Sprachlehre, c'est une approche plus abstraite de la diversité des langues qui est alors proposée ${ }^{41}$. L'élève est capable de décomposer la forme verbale en ses éléments constitutifs (par exemple, dans le cas de l'allemand: le pronom personnel, le verbe d'action, l'auxiliaire). En approfondissant ce mouvement d'analyse, il arrive aux langues dépourvues de flexion, comme le chinois; d'où il peut ensuite remonter, par la synthèse, à des formes verbales complexes.

38. Voir les indications biographiques dans GS I.1, p. 239, et GS III, p. 851. Au sujet de cet essai, voir notamment Myriam Bienenstock, « Assimilation - Dissimilation. Rosenzweig sur l'école », in Myriam Bienenstock (éd.), Héritages de Franz Rosenzweig, op. cit., p. 140-148.

39. GS III, p. 383.

40. Ibid.

41. GS III, p. 399. 
Rosenzweig suggère que la connaissance des langues vaut également initiation aux principales opérations de l'esprit : la pratique des langues rend compte de la « vie de l'esprit humain, en particulier sa vie logique », qui est à la fois ancrée dans un point de départ naturel - le sol des langues - et tendue vers le ciel de la connaissance objective ${ }^{42}$.

On peut donc formuler ainsi la version linguistique de la thèse de l'unité du langage : par-delà la diversité empirique des langues, il existe pour elles toutes une grammaire universelle, et la comparaison des formes de chaque langue permet de faire l'expérience de l'unité profonde du langage.

Rosenzweig, en insistant sur la nécessité d'une bonne connaissance des langues en leur diversité, redonne à la grammaire scolaire (Schulgrammatik) toute son importance. Parler de grammaire philosophique n'implique pas de placer la grammaire sous la coupe de la philosophie, comme en témoigne le texte qui donne son titre à notre étude. Cette lettre d'octobre 1917 à Eugen Rosenstock-Huessy est importante à plus d'un titre, car elle livre la première formulation de la théorie rosenzweigienne des personnes grammaticales: «mon Je surgit dans le $\mathrm{Tu}$ » (mein Ich entsteht im $\mathrm{Du}$ ). On reconnaît là un thème cher à Rosenstock-Huessy, pour qui ce n'est pas le Je, mais le Tu, qui est premier. Dans son essai Angewandte Seelenkunde, dont le brouillon avait été communiqué dès 1916 à Rosenzweig, il appelle de ses vœux une grammaire bien comprise de l'âme humaine, qui reconnaisse cette priorité du Tu et montre comment l'âme s'éveille en l'être humain lorsqu'on s'adresse à lui. C'est pourquoi Rosenstock-Huessy s'en prend avec virulence à la grammaire enseignée à l'école : non seulement celle-ci ne présente que la surface des faits grammaticaux qui constituent en profondeur la grammaire de l'âme ${ }^{43}$, mais elle conduit en outre à des intuitions philosophiques erronées. En nous faisant apprendre les conjugaisons dans un certain ordre (amo, amas, amat...), en choisissant la première personne du singulier comme forme de référence du verbe, la grammaire alexandrine impose la prépondérance de cette première personne et conduit au préjugé d'un primat du Je dans le réel comme dans le langage ${ }^{44}$. La philosophie grammaticale doit rompre avec les habitudes de la grammaire scolaire si elle veut remplir la tâche qui est la sienne : être l'instrument d'une connaissance de l'âme humaine $e^{45}$.

42. GS III, p. 402. Une idée similaire figure chez Hegel dans le «Discours du Gymnase» du 29 septembre 1809. Voir G.W.F. Hegel, Textes pédagogiques, trad. Bernard Bourgeois, Paris, Vrin, 1990, p. 85 : «La grammaire a, en effet, pour contenu, les catégories, les productions et déterminations propres de l'entendement: c'est donc en elle que l'on commence à apprendre l'entendement lui-même ».

43. Eugen Rosenstock-Huessy, Die Sprache des Menschengeschlechts. Eine leibhaftige Grammatik in vier Teilen, Heidelberg : Schneider, 1963-1964, p. 757 : « Die Schulgrammatik mit ihren Konjugationslisten photographiert die Oberfläche, das Nebeneinander der sprachlichen Erscheinungswelt». La même critique vaut d'ailleurs de la philosophie du langage, qui ne saurait être qu'une philosophie de la surface.

44. Ibid., p. 754.

45. Ibid., p. 752. 
S'il reprend le principe d'une philosophie grammaticale, Rosenzweig refuse que cette méthode nouvelle fasse peser des exigences trop élevées sur les grammaires des langues naturelles. Comme Rosenstock-Huessy devrait le savoir, puisqu'il a lui aussi lu l'ouvrage de Finck, bien des langues ont d'autres manières d'exprimer la relation entre le sujet et le verbe : on ne peut donc pas reprocher à toute grammaire de mettre l'accent sur la première personne du singulier.

Allons plus loin : à la vérité, tu n'es pas du tout aussi finckifié [verfinckt] que tu le prétends. Si c'était le cas, tu penserais aux 234 ou 169 ou je ne sais combien de langues qui ne disent pas «cogito» [je pense], mais « cogitaremeum » [mon penser] [...] - Parmi les 321 langues de Finck, y en at-il une qui fonctionne tout à fait sans Je et Tu, en se contentant par exemple d'un système de la troisième personne, où l'on progresse par degrés du proche au lointain (celui-ci, celui-lád ${ }^{46}$, où donc le Je ne serait caractérisé que comme le plus proche, et le Tu comme le plus proche parmi ce qui est lointain? Philosophus non supra grammaticam, mais pourquoi ne devrait-il pas y avoir de grammaires antiphilosophiques ${ }^{47}$ ?

Cette expression signale la volonté de protéger la diversité des langues des critiques du philosophe. Il ne s'agit pas seulement de rappeler la pluralité empirique des langues (peu importe qu'il en existe « 234 ou 169 ou je ne sais combien »), mais de souligner la diversité qui affecte essentiellement le langage. Le langage humain, c'est le fait que les choses peuvent se dire de plusieurs manières. S'il est possible qu'il existe des grammaires antiphilosophiques, c'est-à-dire dont les formes semblent s'opposer à tel ou tel principe philosophique, cela veut dire que la philosophie doit se mettre à l'écoute de la grammaticité du langage, et non de la grammaire de telle ou telle langue particulière. À ce titre, la grammaire philosophique de Rosenzweig est bien Sprachlehre - théorie du langage prenant en compte à la fois la « lettre » (les langues en leur diversité), «l'esprit » (l'essence du langage) et le « logos » (les facultés de l'esprit humain) -, là où RosenstockHuessy en reste à une Grammatik, sous la forme d'une "grammaire de l'âme » dont il espère tirer une méthode d'analyse applicable aux sciences sociales.

L'Étoile de la Rédemption (dont, aux dires de son auteur, la rédaction put commencer en 1918 lorsque les "pensées sur le langage » mûries depuis deux ans eurent trouvé leur ordre ${ }^{48}$ ) nous semble être la réponse complète de Rosenzweig à cette divergence méthodologique avec son correspondant. Prenant acte du passage de la lettre à l'esprit du langage, l'Étoile tient lieu en

46. En français dans le texte.

47. GS I.1, p. 471-472 (ma traduction). Dans l'Étoile, Rosenzweig fait également allusion aux langues ergatives (comme le basque), qui insistent selon lui sur le processus décrit par le verbe et non sur le sujet et l'objet en tant que tels : " Au lieu de dire : "La cigogne avala la grenouille", bien des langues disent : "Il y eut avaler de la part de la cigogne en rapport avec la grenouille" » (Étoile p. 188, Stern p. 144).

48. Gritli-Briefe, p. 144. 
quelque sorte de Sprachlehre pour le programme scolaire tracé dans l'essai de 1916. C'est pourquoi l'étude des langues au moyen de la grammaire comparée n'est pas développée dans l'Étoile: cette tâche doit être prise en charge ailleurs, dans l'institution scolaire.

Cependant, l'Étoile enrichit également ce projet d'une dimension théologique. L'unité du langage y est affirmée en un sens qui s'oppose non seulement à la pure diversité empirique des langues, mais également à la séparation entre le langage de l'homme et le langage de Dieu. Dans l'exégèse du Cantique des Cantiques, qui constitue "l'analyse grammaticale » par laquelle se clôt le chapitre sur la Révélation, Rosenzweig affirme que Dieu et l'homme partagent une même langue et que la parole de l'homme peut être cherchée dans la parole de Dieu; c'est à cette seule condition que la Révélation pourra être véritablement comprise comme un dialogue $e^{49}$.

Le philosophe ne peut ignorer le fait que cette parole de Dieu nous a été transmise sous la forme d'un texte rédigé dans une langue particulière, l'hébreu. Faut-il dès lors penser que la «langue sainte», comme Rosenzweig l'appelle dans l'Étoile, est la « langue une » qui donnerait à tout le langage son unité par-delà la diversité des langues ? C'est l'hypothèse que fait Jean-Luc Évard, traducteur des essais que Rosenzweig a consacrés dans les années 1920 à la langue hébraïque et à la traduction de la Bible. Voyant dans la pensée rosenzweigienne de l'hébreu un «théologoumène », il conclut à l'existence d'un conflit entre "Rosenzweig traducteur», très attaché à la diversité des langues, et «Rosenzweig théologien », convaincu que tout le langage est dans la Bible et que toutes les langues sont dans $1^{\prime}$ hébreu $^{50}$. À nos yeux, pourtant, il n'y a pas là de conflit. La langue hébraïque ne se substitue pas au motif de l'unité du langage, qui ne saurait être ramené à telle ou telle langue particulière, mais qui concerne ce que l'essai de 1916 appelait l'essence du langage. Il est vrai que la réflexion sur l'hébreu du texte biblique a bien un rôle à jouer: elle est peut-être une manière, pour Rosenzweig, de prendre position sur la question de l'origine des langues, à laquelle la thèse de l'unité du langage semble mener en droite ligne. Sa réponse est conforme à celle des linguistes de son temps. S'il semble admettre l'hypothèse indo-européenne ${ }^{51}$, il n'assigne jamais

49. Étoile p. 281, Stern p. 221. - Voir Inken Rühle, Gott spricht die Sprache der Menschen. Franz Rosenzweig als jüdischer Theologe: eine Einführung, Tübingen: Bilam, 2004.

50. Jean-Luc Évard, «Les noms de la parole : Franz Rosenzweig traducteur », in L'Écriture, le verbe et autres essais, op. cit., p. 11 et p. 16.

51. Rosenzweig emploie le terme « indo-germanique », comme c'était l'usage en son temps. Dans "Volksschule und Reichsschule », il paraît souscrire à l'hypothèse d'une origine indienne de la civilisation européenne à travers la Grèce et l'Allemagne ; le concept européen de temps, par exemple, n'a pu pousser que sur un sol indo-germanique, « des Indiens à Kant en passant par Platon, Augustin et Eckhart» (GS III, 401). Sa position est cependant nuancée et s'oppose à l'hypothèse d'une détermination de la pensée, donc de la culture, par la langue. Sur l'hypothèse indo-européenne et ses conséquences idéologiques, voir Maurice Olender, Les Langues du Paradis. Aryens et Sémites : un couple providentiel, Paris, Gallimard/Seuil, « Hautes Études », 1989. 
d'origine unique aux langues du monde. Pas plus le sanskrit que l'hébreu ne sont pour lui la langue originelle de l'humanité. L'assignation d'une telle origine relèverait sans doute de ce que Rosenzweig dénonce sous le nom de " philosophie naturelle du langage $»^{52}$ : une confusion entre la dimension anthropologique du langage, marquée par la différence entre les ethnè, et sa dimension théologique, vouée à l'unité.

À cette Naturphilosophie der Sprache, Rosenzweig oppose donc une philosophie de la Révélation. Mais celle-ci est nécessairement tournée vers l'avènement d'une langue de la Rédemption: "La Révélation elle-même parle une langue qui "n'est pas encore là", qui deviendra langue de l'homme seulement lorsque la plénitude des ethnè sera arrivée (cf. le prophète : "je donnerai aux peuples une lèvre pure $\left.{ }^{53 ")}\right)$. Ainsi, comme le confirme l'Étoile, la manière la plus juste de formuler la thèse de l'unité du langage dans sa version théologique est de la comprendre comme une langue à venir : la diversité des langues est le signe que la Rédemption n'est pas encore réalisée, mais lorsque le temps sera venu, l'unité essentielle du langage apparaîtra sous la forme d'une langue de l'humanité ${ }^{54}$.

La thèse de l'unité du langage est donc marquée, dans ses deux formulations, par une dimension utopique : rêve d'un apprentissage complet des langues humaines, espérance messianique d'une langue unique de l'humanité. Mais Rosenzweig pense aussi que, dans le temps présent, une approximation de la connaissance des langues humaines et une anticipation de la langue unique sont possibles à travers la traduction. Pour les hommes du temps de la Révélation, l'unité du langage humain a une signification immédiate: elle signifie que les langues sont traduisibles. La traduction nous permet de faire l'expérience de cette unité profonde, elle anticipe l'avènement du «Jour de concorde» des langues, "qui ne peut surgir que dans chacune des langues, non pas dans l'espace vide "entre" elles $»{ }^{55}$. Si cette lecture est correcte, cela signifie que la grammaire a vocation à se prolonger en une théorie et une pratique de la traduction, lieu de la Rédemption des langues mais aussi aboutissement de toute philosophie du langage.

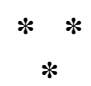

À la lumière de ces éléments, nous concluons que le grand œuvre de Rosenzweig est théorie du langage en un triple sens : contribution moderne au projet classique de grammaire philosophique; achèvement d'un projet

52. Voir la lettre à Eugen Rosenstock-Huessy de mars 1917, Gritli-Briefe p. 3-4.

53. Ibid., p. 3. L'allusion à la « lèvre pure » est tirée de Sophonie 3, 9.

54. Étoile p. 162, Stern p. 122.

55. Franz Rosenzweig, L'Écriture, le verbe et autres essais, op. cit., p. 157. 
pédagogique original ; théologie du langage et de la parole de Dieu. Il faut souligner le caractère paradoxal d'une grammaire philosophique qui repose sur une lecture théologique de certains faits de langue, par ailleurs étudiés au moyen des outils de la linguistique. Dans son projet grammatical tel qu'il s'est formulé en 1916-1917, Rosenzweig combine les apports de la tradition de théorie générale du langage et d'une linguistique comparative d'inspiration humboldtienne. Le principe de l'unité du langage est la thèse qui rend possible cette grammaire; dans l'Étoile et les textes ultérieurs, il offre aussi un motif à l'espérance, un principe herméneutique à la lecture du texte biblique, et une motivation à la tâche de la traduction.

Comme le rappelle l'essai de 1925 «La pensée nouvelle », l'ensemble de cette réflexion doit pourtant se comprendre encore dans un cadre philosophique. Le projet grammatical trouve son point de départ dans la critique de l'idéalisme allemand et débouche sur le projet d'une refondation théologique de la philosophie. À cette ambition, il est sans doute légitime d'adresser la question suivante, comme Rosenzweig le fait lui-même dès l'Étoile: «Est-ce encore de la science ? ${ }^{56}$

L'expression de grammaire philosophique entraîne bien sûr la tentation d'un rapprochement avec les projets de Husserl ou de Wittgenstein. Hilary Putnam, Paul Franks et Cora Diamond ont évoqué une proximité entre Rosenzweig et Wittgenstein sur d'autres thèmes ${ }^{57}$, mais à notre connaissance, la question de la grammaire reste encore à explorer. Si la grammaire est liée chez les deux auteurs à une préoccupation thérapeutique (en particulier dans le Livret sur l'entendement sain et malsain, un texte que Rosenzweig ne destinait pas à la publication), les divergences ne manquent pas : au motif rosenzweigien de l'unité du langage répond chez Wittgenstein la méfiance envers toute approche trop unitaire du phénomène linguistique et l'insistance sur la diversité des jeux de langage. L'orientation théologique de la pensée de Rosenzweig semble également marquer une différence majeure d'avec la grammaire au sens wittgensteinien. Cependant, que Wittgenstein ait pu évoquer, au détour des Recherches philosophiques, "la théologie comme grammaire ${ }^{58}$ doit faire envisager l'hypothèse d'une influence

\section{6. Étoile p. 154-155, Stern p. 117.}

57. Hilary Putnam, Jewish Philosophy as a Guide to Life. Rosenzweig, Buber, Levinas, Wittgenstein, Bloomington and Indianapolis, Indiana University Press, 2008 ; Paul Franks, "Everyday Speech and Revelatory Speech in Rosenzweig and Wittgenstein », Philosophy Today, Spring 2006, 50, 1, p. 24-39 ; Cora Diamond, "Croyance, compréhension et incompréhension: Wittgenstein et la religion », ThéoRèmes [En ligne], 1|2011, mis en ligne le 15 décembre 2011, consulté le 6 novembre 2017.

58. Ludwig Wittgenstein, Recherches philosophiques, trad. F. Dastur, M. Élie, J.-L. Gautero, D. Janicaud et É. Rigal, Paris, Gallimard, 2014, p. 171 (§373). L'allusion se trouve également dans les cours de 1933 à Cambridge, où la référence à Luther est explicitée. Voir Alice Ambrose (éd.), Wittgenstein's Lectures, Cambridge, 1932-1935: From the Notes of Alice Ambrose and Margaret Macdonald, Amherst, NY: Prometheus Books, 2001, p. 32; D. G. Stern, B. Rogers et G. Citron (éd.), Wittgenstein, Lectures, Cambridge 1930-1933. From the Notes of G. E. Moore, Cambridge, Cambridge University Press, 2016, p. 320-321. 
commune aux deux auteurs: celle de Luther, principal interlocuteur de Rosenzweig dans sa réflexion sur la traduction, et autre grand critique de la philosophie depuis le point de vue d'une grammaire considérée comme l'outil de la compréhension de la Parole ${ }^{59}$.

59. Voir Philippe Büttgen, Luther et la philosophie. Études d'histoire, Paris, Vrin, 2011, en particulier chapitre III, p. 87-121. Sur la relation de Rosenzweig à Luther, voir en particulier " L'Écriture et Luther », in L'Écriture, le verbe et autres essais, op. cit., p. 55-82. 